\title{
RESPIRATORY CRISIS AFTER THYMECTOMY IN PATIENTS WITH MYASTHENIA GRAVIS
}

\author{
BY \\ GEORGE W. PENNINGTON* AND F. RONALD EDWARDS \\ From the Department of Pharmacology and General Therapeutics, Liverpool University, and the Thoracic \\ Unit, Broadgreen Hospital, Liverpool
}

(RECEIVED FOR PUBlication JANUARY 3, 1960)

The term "crisis," as applied to myasthenia gravis, usually implies failure of the muscles concerned with respiration, obstruction of the airway due to weakness of the laryngeal and pharyngeal muscles, and the accumulation of salivary and bronchial secretions. It is in these circumstances that apparent resistance to antimyasthenic drugs sometimes develops.

In recent years, thymectomy has been established as a means of alleviating the signs and symptoms of myasthenia gravis. The operation has sometimes been followed by a period of respiratory crisis which has proved difficult to control.

The present paper deals with the onset and treatment of crisis as it has occurred in four out of 10 patients subjected to thymectomy in the Thoracic Unit of Broadgreen Hospital, Liverpool.

\section{CASE RePorts}

CASE 1.-A woman, aged 35, had a 10-month history of weakness affecting the external ocular muscles, the muscles of deglutition, and the small muscles of the hands. She was moderately well controlled with pyridostigmine bromide (mestinon), $300 \mathrm{mg}$., two hourly, orally. A total thymectomy was carried out and an adenoma of the thyroid gland was removed. The thymus weighed $32 \mathrm{~g}$., histologically exhibiting numerous active germinal centres. Immediately after operation her condition was satisfactory with an adequate respiratory exchange. Medication was continued with neostigmine bromide, $2.5 \mathrm{mg}$. intramuscularly at threehourly intervals. Atropine was administered to counteract the muscarinic effects of the anticholinesterase drug, while analgesia was maintained by the administration of $25 \mathrm{mg}$. pethidine at four-hourly intervals. Sixteen hours after operation she suddenly became extremely dyspnoeic, but eventually her shallow respiration became deeper and she recovered. She experienced further difficulty with respiration five hours later, during which she became cyanosed; endotracheal intubation, and

* Present address: Department of Pharmacology, University of California Medical Center, Los Angeles 24, California. later tracheotomy and assisted respiration were necessary. This had to be continued for the next 10 days before $N$ she again began to breathe spontaneously. During this $ᄋ$ period a number of tensilon tests were carried out to try to ascertain the type of crisis but with equivocal os results. Medication was stopped for 24 hours without $\frac{\mathbb{}}{0}$ improvement, after which it was again resumed. Achro- $\overrightarrow{\mathbb{D}}$ mycin, $250 \mathrm{mg}$., was given six hourly prophylactically. On the sixteenth post-operative day, respiration was $\underset{\mathbb{C}}{-}$ sufficiently well established to justify removal of the $\vec{P}$ tracheotomy tube. She progressed satisfactorily, $\mathscr{\odot}$ receiving as medication $45 \mathrm{mg}$. of neostigmine, two ? hourly during the day, and $30 \mathrm{mg}$. two hourly at nighg. On the twenty-second day after operation, she developed an upper respiratory tract infection, which resulted i the all-round accentuation of myasthenic symptom During the ensuing six days she produced large quantities $\frac{0}{8}$ of sputum; a throat swab culture showed monilia $\unrhd$ and coliform bacilli. On the twenty-eighth day she $\overrightarrow{\vec{P}}$ again collapsed, became extremely cyanotic, and then $\frac{3}{3}$ unconscious. An endotracheal tube was passed, and she regained consciousness. Two hours later apnoea recurred, and it was necessary to re-establish the tracheotomy and to give the patient assisted respiration. Her condition continued to deteriorate and the peri- $\frac{0}{2}$ pheral circulation remained collapsed. With the $x$ continuance of artificial respiration, however, she rallied $\frac{5}{3}$ and later radiographic and clinical examination revealed that the right lung had collapsed.

At the thirty-sixth day she began to improve clinically so that by the forty-eighth day she was progressing 0 satisfactorily. A new régime of medication was established, consisting of pyridostigmine bromide, $240 \mathrm{mg}$. $\frac{7}{0}$ three hourly by mouth, and neostigmine, $2.5 \mathrm{ml}$. subcutaneously three times daily.

On the fifty-first post-operative day she suddenly collapsed, with marked weakness of all voluntary musculature. The results of numerous tensilon tests $\mathrm{\omega}$ to ascertain the nature of the crisis were equivocal, but treatment was discontinued for 24 hours on the assumption that it was cholinergic in nature. Little change was noted at the end of this period, when pyridostigmine of bromide, $120 \mathrm{mg}$. two hourly, was reintroduced. Throughout the period of crisis the electrolyte picture was corrected as abnormalities appeared. 
During the following 14 days, respiration had to be assisted. On the sixty-fifth post-operative day she started to improve, and this improvement continued so that at the ninetieth day it was possible to remove the tracheotomy tube. It is now three and a half years since the time of the operation, during which period this improvement has continued. She is able to lead a fairly normal family life, and, as an extremely intelligent woman, adjusts her own pyridostigmine requirements according to her clinical condition. This dosage varies between 12 and 20 tablets (60 mg.) daily.

CASE 2.-A woman, aged 43, had a history of diplopia, dysarthria, dysphagia, and generalized weakness for one year. Respiratory embarrassment occurred often, particularly at the times of any concomitant upper respiratory tract infection.

The thymus was removed and weighed $22 \mathrm{~g}$. There was a small tumour in one lobe. Histological section revealed that this was a benign thymoma, while the thymus tissue displayed the germinal lymph follicles which are characteristically found in myasthenia gravis. With the knowledge of the respiratory difficulty experienced in the previous patient, an elective tracheotomy was performed at the time of the operation. On the first post-operative day frequent suction was required in order to remove profuse salivary secretion. Her general condition deteriorated, so that by the second post-operative day, due to her cyanotic appearance, continuous artificial respiration was required. This was maintained until the eighth day, by which time she was having periods of spontaneous breathing. Treatment throughout this period consisted of oral pyridostigmine bromide, $240 \mathrm{mg}$. six hourly, together with atropine, grains $1 / 100$, as required. Achromycin, $250 \mathrm{mg}$., six hourly was given prophylactically.

On the tenth post-operative day she breathed spontaneously for 12 hours, while by the twelfth day respiration was sufficiently satisfactory for the tracheotomy tube to be removed. This improvement continued so that by the twentieth day the myasthenic condition was so much better that she was talking and swallowing in a normal manner. A close watch was kept on the electrolyte picture throughout the post-operative period.

Her further progress was delayed by the clinical and radiographic finding of a left-sided pleural effusion. Aspiration of the chest on two occasions resulted in the removal of $35 \mathrm{oz}$. and $3 \mathrm{oz}$. of fluid; both specimens were sterile on culture. Thereafter, she gradually improved, so that by the twenty-eighth day she was up, walking about the ward.

It is now 18 months since the operation; the myasthenic condition has improved markedly, and, although she still takes 20 tablets of pyridostigmine daily, she is able to carry out the normal duties of a housewife as well as being employed part time.

CASE 3.-A woman of 58 years, with a two-year history of excessive fatigability, ptosis, and dysphagia, was maintained on 16 tablets of pyridostigmine bromide daily.
At operation, the gland weighed $15.5 \mathrm{~g}$. and there was a small tumour situated in the left upper pole of the gland. Histological examination revealed a mixed pattern of epithelial and lymphoid elements, while the tumour was identified as an encapsulated benign thymoma. An elective tracheotomy was again undertaken at the time of operation, and, in addition, the patient was given chloramphenicol, $250 \mathrm{mg}$. eight hourly.

There were no untoward occurrences until the fourth post-operative day, when she had a short period of apnoea followed by a period of rapid, shallow respiration. A " tensilon test" (edrophonium hydrochloride) carried out at this time resulted in an improvement in respiration, indicating that the anticholinesterase medication she was receiving should be increased. Despite this, respiration deteriorated and two hours later artificial respiration was necessary. A bronchoscopy was performed and a large amount of inspissated secretion was removed from the right bronchus. For a time her general condition improved following this procedure, and, on the assumption that the belladonna which she was receiving to counteract the muscarinic effects of pyridostigmine had contributed to the formation of the inspissated mass, treatment with it was discontinued.

Ten hours later, she lost consciousness and artificial respiration was again necessary. Thereafter intermittent artificial respiration was necessary with gradual improvement, until, on the eleventh day, the tracheotomy tube was removed. In the meantime belladonna was added to the four-hourly dosage of pyridostigmine bromide (one tablet) she was receiving because she had profuse diarrhoea. Her general condition was now much improved, although there were moist sounds in the chest detected by auscultation. Radiographic examination confirmed the presence of a right-sided pleural effusion, which was aspirated, $11 \mathrm{oz}$. of blood-stained serous fluid being removed.

Shortly afterwards she was discharged from hospital, the pyridostigmine tablets, by this time, having been reduced to two per day.

CASE 4.-A child of 13 years had a six-month history of diplopia, ptosis, and generalized weakness, rapidly becoming more severe. Combined neostigmine and pyridostigmine therapy was not adequately controlling the myasthenic condition.

At operation a large thymus gland (40 g.) was found, which, when examined histologically, revealed considerable lymphoid hyperplasia.

In accordance with previous experience an elective tracheotomy was performed and achromycin, $250 \mathrm{mg}$. six hourly, was administered prophylactically.

Immediately after operation there was no respiratory difficulty, but 24 hours later it was found necessary to give assisted respiration. Neostigmine was given subcutaneously but produced no clinical improvement. Artificial respiration was continued until the sixth day when some improvement in her general condition resulted in periods of spontaneous respiration. 
On the ninth post-operative day, she suddenly became cyanosed and dyspnoeic, requiring continuous artificial respiration. The medication she was receiving, pyridostigmine tablets, one four hourly, was increased to four tablets four hourly, but this resulted in little change in her condition, and was discontinued. Her general condition deteriorated during the tenth and eleventh post-operative days. As supportive therapy for low blood pressure, $50 \mathrm{mg}$. hydrocortisone was given with some improvement.

On the evening of the eleventh day she became comatose and, despite the use of central stimulants, she died. A post-mortem examination revealed the presence of bronchopneumonia, but nothing else as the cause of death.

\section{Discussion}

Crisis following thymectomy does not occur in all cases. The cases quoted here are those in which crisis, to a lesser or greater degree, did occur. Keynes (1949), who has had considerable experience of thymectomy and its complication, is of the opinion that death following thymectomy may in all instances be attributed to pulmonary complications. These complications are due either to an acute myasthenic crisis in which the patients drown in their own secretions, to atelectasis of part of a lung, or to a flare-up of a pre-existing bronchitis. The first three cases described in this paper would certainly partially substantiate this statement, gross pulmonary lesions occurring in each. Keynes (1949) also emphasizes that death may be due in some cases to failure of both heart and respiration, and quotes a case in support of this statement. Osserman (1959) in America agrees that failure of the heart and respiration occurs, and that it should be listed as a cause of death, even though the cause of this fa lure is not known. The death described in Case 4 could be placed into this category.

From experience, the elective performance of a tracheotomy should be considered in all cases of thymectomy undertaken for myasthenia gravis. Its presence considerably aids the continuance of respiration should a crisis occur, and avoids any undue delay in establishing immediate assisted respiration. Post-operatively many myasthenic patients have a poor cough reflex, which increases the difficulty of removing bronchial secretions. The tracheotomy tube permits the frequent passage of sterile catheters for aspiration of secretions. It is essential that the sterility of the catheters be meticulously maintained as bronchial infection very easily occurs. It is in an attempt to avoid infection that one of the tetracycline group of drugs has been used prophylactically in the later cases. Before this therapy was initiated, penicillin and strepto- mycin were given until the organisms had been cultured and sensitivity tested, as described by Tether (1955), who believes this to be the essential part of the treatment of crisis. It was, however, thought better to try to avoid infection rather than to treat it after it had occurred.

It is often difficult under the conditions described to differentiate between a myasthenic and a cholinergic crisis. The "tensilon" test is often equivocal, and little help may be gained from it. Randt (1953) believes that a patient in crisis who does not respond to several doses of neostigmine, and is unable to maintain proper pulmonary ventilation, should have all anticholinesterase medication withdrawn and respiration maintained artificially. This was done in the first case, but the lack of improvement after 24 hours made it appear unlikely that the crisis was cholinergic in origin. Viets (1954) agrees that the withdrawal of treatment is justified as the best way to handle a desperate situation, although Schwab (1958) and Grob (1958) warn that withdrawal of specific therapy is fraught with hazards and makes the nursing care much more difficult. Nevertheless Eaton and Clagett (1955), who have had considerable experience in the treatment of crisis, point out that they have found no substitute for tracheotomy, use of the respirator, and mechanical forms of respiration.

Good nursing care in a competent respiratory unit is probably the greatest single factor in overcoming the crisis; the nursing staff should be experienced in the care of artificially respired patients, and be fully acquainted with the apparatus needed to perform this function.

In two of the cases described here, tumours of the thymus gland were present. In neither were they recognized before operation, despite intensive radiographic investigation. Keynes (1949) believes that the presence of a tumour adds to the technical difficulty of removal of the thymus gland, and that often a short time after operation the patient tends to exhibit respiratory symptoms which may become irreversible. In Cases 2 and 3 tumours were found at operation, and, particularly in the former, the post-operative phase was stormy. It is impossible to say, however, if this was due to the presence of the tumour, as Case 1, where no tumour was found, also went through a most critical postoperative phase.

When crisis occurs following operation, the outcome depends upon the close co-operation of the surgeon, physician, anaesthetist, and nursing staff. It is only by the mutual knowledge of the part that each has to play that the crisis can be 
overcome and the danger of over-treatment minimized. It is thus of advantage that such patients should be operated upon in a unit where the treatment of respiratory arrest is understood and where a skilled team is present.

\section{SUMMARY}

The management of crisis following thymectomy in four myasthenic patients is described.

Elective tracheotomy and prophylactic antibiotic therapy are recommended.

Various procedures are discussed which may prove necessary during the post-operative phase.

The mutual co-operation of surgeon, physician, anaesthetist, and nursing staff is of such value in these cases that the treatment is best undertaken where a respiratory paralysis unit is already established.

We would like to thank Professor A. Wilson, of the Department of Pharmacology and General Therapeutics, Liverpool University, for his interest and valuable co-operation during the writing of this paper.

\section{REFERENCES}

Eaton, L. M., and Clagett, O. T. (1955). Amer. J. Med., 19, 708.

Grob, D. (1958). Personal communication; quoted in Myasthenia Gravis, by Osserman, K. E. Grune and Stratton, New York and London.

Keynes, G. (1949). Brit. med. J., $2,611$.

Osserman K. (1959). Personal communication.

Randt, C. T. (1953). Med. Clin. N. Amer., 37, 535.

Schwab, R.S.(1958). Personal communication; quoted in Mvasthenia Gravis, by Osserman, K. E. Grune and Stratton, New York and London.

Tether, J. E. (1955). Amer. J. Med., 19, 740.

Viets, H. R. (1954). New Engl. J. Med., 251, 97, 141. 